\title{
RESOLUCIÓN DE PROBLEMAS EN LA ENSEÑANZA DE LA GEOLOGÍA: CONTRIBUCIONES DE UN ESTUDIO EXPLORATORIO
}

\author{
S. Carrasquinho ${ }^{(1)}$, C. Vasconcelos $^{(2)}$ y N. Costa ${ }^{(3)}$ \\ ${ }^{(1)}$ Escuela Básica de 20 y 30 Ciclos de Revelhe, Fafe. (Portugal) \\ ${ }^{(2)}$ Departamento de Geología. Facultad de Ciencias/Universidad De Oporto (Portugal) \\ ${ }^{(3)}$ Departamento de Didáctica y Tecnología Educativa/Universidad De Aveiro (Portugal)
}

[Recibido en Mayo de 2006, aceptado en Septiembre de 2006]

\begin{abstract}
RESUMEN ${ }^{\text {(Inglés) }}$
Esta investigación consistió en el fundamento, concepción, aplicación y evaluación de una planificación centrada en enseñar un tema de Geología, a través de I resolución de problemas. La planificación se efectuó en el contexto natural del aula, en la asignatura de Ciencias Naturales, en una escuela del Norte de Portugal con alumnos de edades comprendidas entre los 12 y 13 años, donde la profesora del grupo asumió el doble papel de profesora e investigadora. Se acudió a una metodología de investigaciónacción, donde se optó por técnicas de recogida de datos como fueron las fichas de trabajo suministradas a los alumnos (por ejemplo, ficha de diagnóstico de concepciones previas sobre el tema), los diarios de clase de la profesora y los snapshots. El estudio permitió comprender la eficacia del proceso de enseñanza y aprendizaje en la optimización de la calidad de la práctica docente, cuando éste: (a) se fundamenta en la investigación en Didáctica de las Ciencias, específicamente en lo que respecta al área de investigación sobre Resolución de Problemas; (b) es dirigido por un profesor que paralelamente asume el papel de investigador y (c) es apoyado por investigadoras con papel activo en el proceso de investigación.
\end{abstract}

Palabras Clave: Resolución de Problemas; Investigación-acción; Diarios de Clase; Snapshot.

\section{INTRODUCCIÓN}

Se trata de un trabajo de investigación llevado a cabo en el curso 2004/2005, en el contexto natural del aula, con alumnos de Ciencias Naturales con edades comprendidas entre los 12 y 13 anos, pertenecientes a una escuela del Norte de Portugal y en el cual la profesora (principal autora de este artículo) asumió el doble papel de profesora e investigadora. En este estudio se realizó una planificación centrada en la enseñanza a través de la resolución de problemas, cuya aplicación tuvo tres propósitos fundamentales: i) la propia formación de la investigadora, principalmente en lo que respecta a la conceptualización y a la práctica de una 
enseñanza a través de la Resolución de Problemas (en este aspecto se deben realzar las dificultades inherentes a la transposición de los resultados de la investigación para la práctica docente, tales como la adaptación de las actividades y la evaluación de la viabilidad de las estrategias definidas); ii) la familiarización de la investigadora con técnicas e instrumentos cualitativos que no son aún muy valorados en la investigación en didáctica de las ciencias (Diarios de Clase y Snapshots), a los cuales se acudió ya sea para suscitar una actitud reflexiva de la profesora, o para conocer el pensamiento de los alumnos (la utilización sistemática de estos instrumentos, durante la planificación, le permitió a la profesora ir reajustando sus estrategias didácticas con el fin de potenciar la calidad en el aprendizaje); iii) contribuir al desarrollo del conocimiento didáctico en lo que respecta a la teorización sobre la práctica docente en el área de resolución de problemas (Evans, 2002).

\section{PLANIFICACIÓN CENTRADA EN LA ENSEÑANZA A TRAVÉS DE RESOLUCIÓn dE PROBLEMAS}

La Resolución de Problemas (RP) puede definirse como un proceso a través del cual el alumno desarrolla una combinación de procedimientos y reglas, cuya aplicación permite solucionar una nueva situación problemática (Angelopoulos y Tsaparlis, 2000) o simplemente como situación ante la cual se está, inicialmente, perdido (Gil Pérez et al, 1999). Actualmente, las principales tendencias en la investigación sobre RP son las de intentar conocer los procesos envueltos en la RP y estudiar modos de enseñar a través de RP (Broekkamp, Taconis; y Ferguson-Hessler, 2001). La enseñanza a través de RP puede permitir desarrollar capacidades muy variadas, tales como capacidades de comunicación y de defensa de diferentes puntos de vista en diversas áreas del conocimiento, en particular de las Ciencias Naturales, así como desarrollar también la capacidad de cooperación en el trabajo de grupo (Gardner, 2003).

La centralidad del concepto de problema en el área de investigación sobre RP, nos llevó a definirlo desde el mismo inicio de nuestro estudio. Aunque existen varias definiciones de problema, en este trabajo se optó por la definición de Lopes (2004, p.37), según el cual, problema es el "acto de demarcación entre lo que un individuo (o comunidad) ya sabe y lo que no sabe, para resolver dificultades o necesidades específicas de conocimiento sentidas por el alumno, para obtener y/o ampliar el conocimiento conceptual y procesal, y desarrollar capacidades cognitivas y afectivas". Así, desde el comienzo de nuestra planificación, nos propusimos ya delimitar lo que los alumnos ya sabían a través de una ficha diagnóstico.

El planteamiento de problemas es fundamental en la enseñanza a través de RP, ésta se define como la actividad que permite formular y distinguir problemas. Según Garret (1995), la mayoría de los profesores cree que la finalidad de la educación, y de la educación científica en particular, es el desarrollo de competencias para identificar y resolver problemas. Esta finalidad ha pasado por la elección de una situación problemática y por la creación de un contexto problemático (Costa, 2002). Podemos considerar la existencia de tres fases en la construcción de un contexto problemático: i) elección por el profesor, o por los alumnos bajo la orientación del profesor, de material informativo $u$ objetos relacionados con acontecimientos naturales $o$ 
tecnológicos; ii) favorecer un ambiente estimulador en el aula para que, bajo la orientación del profesor, los alumnos desempeñen las tareas; iii) debate en pequeños grupos, y con el resto de los grupos del aula, sobre la formulación de preguntas, su pertinencia y la incidencia de las preguntas en los aspectos fundamentales. Así, en nuestro estudio, la información sobre el tema a impartir se obtuvo de Internet y de libros y publicaciones que se encuentran en la biblioteca de la Escuela. En la segunda fase de la construcción de un contexto problemático, Lopes (2004) refiere que es esencial que todos los alumnos tengan acceso a la información, lo cual se verificó atendiendo a la disponibilidad de materiales ofrecidos por el recinto escolar en cuestión. Teniendo en cuenta la tercera fase de la construcción de un contexto problemático, la profesora promovió situaciones de debate siempre que las actividades dinamizadas así lo permitían.

Neto (1995), Gardner (2003) y Lopes (2004) defienden que la enseñanza a través de $\mathrm{RP}$ engloba varios procesos y etapas. En este trabajo se tuvieron en cuenta las seis dimensiones definidas por Lopes (2004): i) identificar o reconocer la existencia del problema y hacerlo suyo (el alumno debe sentir que el problema le atrae y que es interesante y relevante); ii) representar el problema y crear la situación (consiste en idear y proyectar lo que el individuo comprende de la situación abordada en el problema); iii) planear un enfoque (hacer operacionales las representaciones conceptuales particulares y la representación conceptual global); vi) obtener respuestas; v) evaluar los resultados y la resolución y vi) extender el modelo de utilización del conocimiento. Algunos autores, como por ejemplo, Gardner (2003) y Neto (1995), exponen que en la enseñanza a través de Resolución de Problemas no es solamente importante la evaluación realizada por el profesor (para la cual, en nuestro caso, contribuyeron mucho los Diarios de Clase elaborados por la profesora), sino que también destacan el valor educativo de la evaluación efectuada por el propio alumno, reconociendo la metacognición como el factor más importante para la calidad y eficacia en la calidad de los aprendizajes y de su transferencia para nuevas situaciones (Valente; Salema; Morais e Cruz, 1989). Con los Snapshots, realizados por los alumnos, se tuvo en cuenta ese objetivo.

\section{EJECUCIÓN DE LA PLANIFICACIÓN - TEMA DE LA GEOLOGÍA}

La planificación centrada en la enseñanza a través de RP se llevó a cabo en las unidades temáticas "Consecuencias de la Dinámica Interna de la Tierra" y "Estructura Interna de la Tierra". La primera unidad se orienta hacia la exploración de los testimonios que demuestran la dinámica de la Tierra, realzando los sismos y los volcanes como resultado de la inestabilidad en la litosfera. En relación al vulcanismo los conceptos analizados deben ser: i) la estructura del aparato volcánico (cráter, chimenea volcánica, cámara magmática); las manifestaciones secundarias de vulcanismo (fumarolas, manantiales o aguas termales y géiseres); la relación entre el tipo de erupción (explosiva, efusiva o mixta), el tipo de aparato volcánico y algunas propiedades del magma (viscosidad y fluido). Así, se debe hacer énfasis en los riesgos y los beneficios de la actividad volcánica. El estudio de los riesgos y la protección de las poblaciones, en relación a la actividad sísmica, es la principal preocupación encontrada en las orientaciones curriculares en el estudio de los sismos. Sin embargo, 
y para que los alumnos puedan comprender este riesgo, se recomienda un abordaje de los conceptos de Hipocentro o Foco y Epicentro, y el contacto de los alumnos con las escalas de Mercalli (modificada) y de Richter, relacionándose el concepto de magnitud con la cantidad de energía liberada en el foco sísmico. La referencia al papel de los sismógrafos y la importancia del análisis de los sismogramas son también consideradas indispensables para el estudio de los sismos. También se recomienda el análisis de mapas de isosistas.

La unidad temática referente a la "Estructura Interna de la Tierra" nos presenta las aportaciones de la Ciencia y la Tecnología para el estudio del interior de la Tierra, principalmente el aporte del estudio de los volcanes y de los sismos para el establecimiento de esa estructura. En relación a los modelos de la estructura interna de la Tierra, se sugiere que se haga referencia al modelo que divide a la Tierra en tres capas (Corteza, Manto y Núcleo) y al modelo que divide a la Tierra en cuatro capas (Litosfera, Astenosfera, Mesosfera y Endosfera), destacando sus límites.

Se escogieron estas unidades por el interés que los alumnos generalmente muestran hacia estos temas, especialmente derivado de hechos ocurridos en un pasado reciente, en particular la erupción del Etna en 2001 (Italia), y sismos y el tsunami ocurridos en el sudeste asiático en 2004. Los problemas definidos por los alumnos han sido: "¿cuál es la constitución del interior del planeta?"; "¿cuáles son las consecuencias de la actividad volcánica?" y "¿cómo prevenir las siniestras consecuencias de la actividad sísmica?". Las capacidades, conocimientos y actitudes que han sido desarrolladas en los alumnos son las siguientes:

- El reconocimiento de transformaciones desde el punto de vista químico, biológico y geológico;

- Uso de modelos y de símbolos en la representación de estructuras, de sistemas y de sus transformaciones;

- Explicación de algunos fenómenos biológicos y geológicos, teniendo en cuenta los procesos físicos y químicos;

- La presentación de las explicaciones científicas que implica pensamiento creativo.

- La identificación de modelos subyacente a las explicaciones científicas;

- Promover una actitud de planteamiento de problemas en los alumnos y el respeto por las diversas opiniones.

- Cooperar con los otros en tareas y proyectos comunes.

- Buscar, seleccionar y organizar la información para transformarla en conocimiento.

\section{METODOLOGÍA DE INVESTIGACIÓN}

En este estudio se adoptó una metodología de tipo Investigación-Acción, apoyada en técnicas cualitativas. La Investigación-Acción analiza los cambios, más o menos profundos, que afectan a una situación educativa con participación de las muestras 
relacionadas y de los participantes implicados (Maren, 1996) que, en nuestro caso, incluye a la profesora-investigadora, a los alumnos del grupo que participa en el estudio y las dos investigadoras (segunda y tercera autoras de este artículo) que colaboraron en el proyecto en el cual se inserta este estudio.

Antes de iniciar la puesta en práctica del planeamiento, los alumnos habían hecho una ficha de diagnostica para definir su saber disponible. En la primera sesión de clase, de 90 minutos, se ha promovido una discusión sobre lo vulcanismo en Portugal de modo que los alumnos pudieran formular el problema. Seguidamente, en grupo, los alumnos habían definido el contenido conceptual inherente a este tema que se debía entender para solucionar el problema. En esta dirección, el profesor proporcionó a los alumnos la interpretación de una presentación en PowerPoint y la visualización de una película de una erupción ocurrida en Cabo Verde, en Abril de 1995. Estos recursos han sido acompañados de la elaboración de fichas de trabajo para el uso de los conceptos presentes en la presentación en PowerPoint y la película. Se planteó un trabajo en grupo para que los alumnos pudieran resumir los diversos conceptos y respuestas al problema. Dividieron la clase en cuatro o cinco grupos, de modo que se facilitara el trabajo en la sala de informática y que el profesor dirigiera la investigación, así como la búsqueda de información a través de los sites de Internet y de la bibliografía previamente sugerida.

En una lección posterior, el profesor supervisó y colaboró con los grupos en la organización y la síntesis de la información buscada. Para clarificar dudas posibles en algunos conceptos, el profesor promovió una discusión sobre las definiciones de seísmo, terremoto, y tsunami. Con el propósito de contestar a las necesidades conceptuales de los alumnos, el profesor medió en la realización de las fichas del trabajo y en la interpretación de figuras y de gráficos. Considerando el contenido aprendido en el transcurso de las dos primeras unidades temáticas del planeamiento, el profesor solicitó a los alumnos la elaboración de un modelo de la estructura interna de la tierra. Estos modelos habían sido discutidos en grupo.

Finalmente, con la colaboración de los alumnos y por medio de un mapa conceptual, el profesor resumió el contenido abordado. Los criterios de evaluación de los alumnos han sido diversos, entre ellos, la actitud y la perseverancia de los alumnos, el pensamiento creativo en las fases de trabajo, el trabajo presentado por los diversos grupos y el desarrollo de una prueba escrita.

Para comprender la perspectiva de los alumnos en el proceso de enseñanza y aprendizaje llevado a cabo, así como estudiar el pensamiento didáctico de la profesora relativo a su práctica docente, se acudió a: i) los "Snapshots" aplicados a los alumnos en los últimos 10 minutos de cada clase, y ii) los Diarios de Clase elaborados por la profesora. También se cabe señalar que los datos recogidos, su análisis e interpretación, fueron ampliamente discutidos por las tres autoras de este artículo.

Finalmente debemos señalar que el grupo seleccionado, uno de los grupos de la profesora-investigadora, comprendía 23 alumnos con edades entre los 12 y 13 años, excepto tres alumnos repetidores (dos de 14 años y uno de 15). Mencionar también que la ejecución de la planificación ocurrió en seis clases de 90 minutos cada una. 


\section{Los Snapshots}

La palabra snapshot (Anexo), de origen anglosajón, significa una observación o impresión aislada de un acontecimiento breve o transitorio. En su esencia, el snapshot se asemeja a un simple cuestionario de respuesta breve, que permite que se tenga un acceso rápido al conocimiento imprescindible a cualquier proceso de evaluación. En este estudio, el snapshot constituyó un instrumento de evaluación formativa, atendiendo a que se trata de un medio de obtención sistemática de información sobre lo que el alumno "sabe", "quiere saber" y "aprendió sobre un determinado tema", contribuyendo a optimizar el desempeño del profesor y a proporcionar al alumno una reflexión sobre su proceso de aprendizaje. Este instrumento permite que los alumnos tomen conciencia del modo en que está transcurriendo su propio proceso de aprendizaje. Desde el punto de vista del profesor, los Snapshots pueden ayudar a los alumnos a monitorizar los progresos de los alumnos, suministrándoles informaciones sobre sus aprendizajes. En este sentido, los resultados del análisis de la información obtenida pueden ayudar en la evaluación del desempeño de los profesores, sirviendo de orientación en la toma de decisiones en el aula (Mertler, 1997). La utilización de este instrumento permite contribuir a mejorar el aprendizaje de los alumnos y la práctica docente del profesor.

\section{Los Diarios de Clase}

Se puede acudir a los Diarios de Clase para intentar acceder al pensamiento del profesor. Los Diarios de Clase son informes a través de los que el profesor narra, explica, analiza e interpreta su acción cotidiana y sus experiencias, constituyéndose como espacio narrativo del pensamiento del profesor (Garcia, 1999). Este instrumento de obtención de datos demuestra lo que Zabalza (1994) denomina como dilemas. De acuerdo con este autor, dilema es un "conjunto de situaciones bipolares o multipolares que se presentan al profesor durante su actividad profesional" (Zabalza, 1994, p. 61) y frente a las cuales el profesor tiene que hacer opciones. Al objeto de sintetizar la variedad de Diarios de Clase existentes, Zabalza (1994) identifica y caracteriza, en función del contenido, tres diferentes tipos de Diarios de Clase: i) el diario como organizador estructural de la clase (diarios de horarios) - se presentan como una simple separación de horarios o explicación de la organización y secuencia de las actividades que se realizarán durante la clase; ii) el diario como descripción de las tareas (diarios de tareas) - las tareas que profesores y alumnos realizan dentro del aula desempeñan un papel fundamental en este tipo de diario, y iii) el diario como expresión de las características de los alumnos y de los propios profesores (diarios de sujetos) - son esencialmente expresivos y auto expresivos. Estos tres tipos de diarios no se excluyen mutuamente, existiendo una gran variedad de diarios con características mixtas. Los Diarios de Clase favorecen de forma irrefutable los procesos metacognitivos y de reflexión (Zabalza, 1994). En este sentido, y entendiéndose la enseñanza como una actividad reflexiva, incentivar a una reflexión profunda y crítica de las prácticas del profesor facilita la alteración de las mismas (Vieira, 1993).

Además de los instrumentos referidos para recoger datos, se utilizaron también otros más directamente relacionados con las actividades concretas realizadas durante el proceso de enseñanza y de aprendizaje. 


\section{ANÁLISIS E INTERPRETACIÓN DE LOS RESULTADOS}

El estudio tuvo un abordaje cualitativo, habiéndose sometido los Diarios de clase y los Snapshots a un análisis de contenido. Es a estos dos instrumentos a los cuales se les dará mayor importancia en este artículo.

\section{Análisis e interpretación de los Snapshoțde los alumnos}

En esta sección se analizan las respuestas de los alumnos a los Snapshots. En cada cuadro se presenta la síntesis del análisis de los Snapshots respondidos por los alumnos en el conjunto de las 6 clases. Es fundamental destacar que, en función de cada pregunta en el snapshot (Anexo), los resultados aparecen resumidos en los Cuadros convertidos en dimensiones de análisis. En relación a la primera dimensión "Aspectos más positivos de la clase" (A), los resultados se describen en el Cuadro 1.

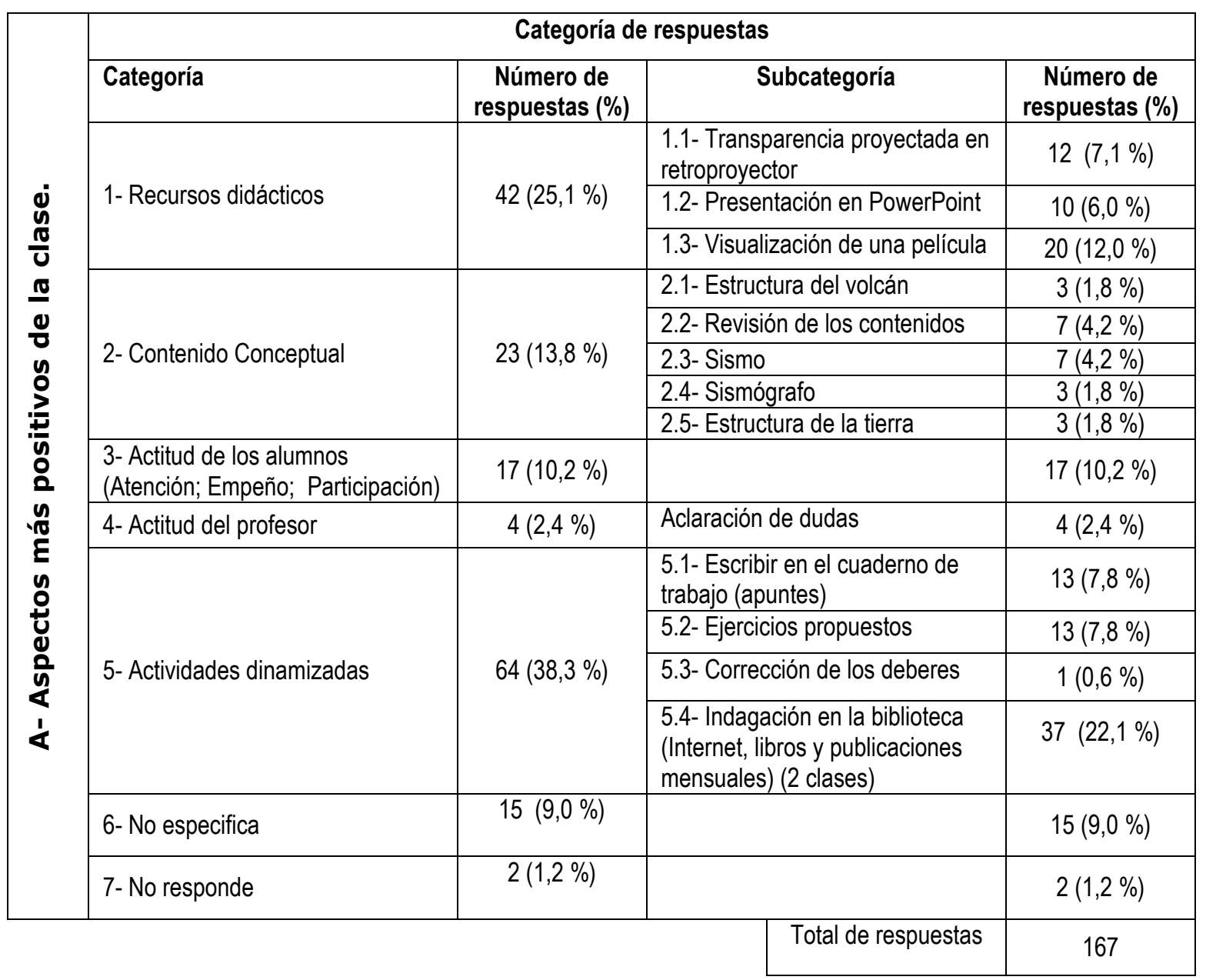

Cuadro 1.- Respuestas de los alumnos en la dimensión A.

Estos resultados muestran la importancia que los alumnos atribuyen a las actividades promovidas por el profesor y a los recursos didácticos utilizados. El elevado porcentaje de respuestas $(22,2 \%)$ que muestra como positiva la búsqueda de información sobre los volcanes en diversas fuentes, refleja el deseo de los alumnos de responder 
autónomamente a la pregunta-problema, y también muestra la motivación para el estudio del tema. La referencia a los recursos didácticos (42 respuestas), principalmente la visualización de la película (20 respuestas), puede estar relacionada con el aspecto lúdico de la tarea donde fue utilizado.

En relación a la dimensión B, "Aspectos menos positivos de la clase", el análisis de las respuestas se encuentra resumido en el Cuadro 2.

\begin{tabular}{|c|c|c|c|c|}
\hline & & Categc & de respuestas & \\
\hline$\frac{\pi}{0}$ & Categoría & $\begin{array}{c}\text { Número de } \\
\text { respuestas (\%) }\end{array}$ & Subcategoría & $\begin{array}{c}\text { Número de } \\
\text { respuestas (\%) }\end{array}$ \\
\hline$\frac{0}{0}$ & 1-Contenidos conceptuales & $5(3,6 \%)$ & $\begin{array}{l}\text { 1.1- Dificultades de comprensión de } \\
\text { contenidos }\end{array}$ & $3(2,2 \%)$ \\
\hline$\geq$ & & & 1.2- Sismos & $2(1,4 \%)$ \\
\hline$\frac{4}{5}$ & $\begin{array}{l}\text { 2- Actitud de alumnos } \\
\text { (Desatención, ruido, } \\
\text { indisciplina) }\end{array}$ & $20(14,4 \%)$ & & $20(14,4 \%)$ \\
\hline$\stackrel{0}{0}$ & & & $\begin{array}{l}\text { 3.1- Escribir en el cuaderno de } \\
\text { trabajos (apuntes, resumen) }\end{array}$ & $9(6,4 \%)$ \\
\hline 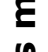 & 3- Actividades dinamizadas & $18(12,9 \%)$ & 3.2- Ejercicios propuestos & $5(3,6 \%)$ \\
\hline & & & 3.3- Indagación (Internet, libros) & $4(2,9 \%)$ \\
\hline 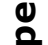 & 4- No especifica (Ninguno) & $39(28,1 \%)$ & - & $39(28,1 \%)$ \\
\hline 4 & 5- No responde & $54(38,8 \%)$ & - & $54(38,8 \%)$ \\
\hline ه' & 6- Respuesta no válida & $3(2,2 \%)$ & - & $3(2,2 \%)$ \\
\hline & & & Total de respuestas & 139 \\
\hline
\end{tabular}

Cuadro 2.- Respuestas de los alumnos en la dimensión B.

El elevado porcentaje de respuestas incluidas en la categoría "No especifica" (28,1\%) y de alumnos que no dan ninguna respuesta "No responde" (38,8 \%) puede deberse a diferentes razones: i) a un cierto recelo de los alumnos de que estos elementos influyan en sus calificaciones y ii) a un ambiente favorable en el aula. Respecto a las actitudes de los alumnos, se observa que 20 de ellos valoran las actitudes de empeño y atención, así como un ambiente organizado en el aula.

En lo que respecta a la dimensión C, "Lo que se aprendió en clase", el Cuadro 3 sintetiza el análisis de los resultados obtenidos.

Es importante destacar la relevancia que los alumnos dieron a los contenidos conceptuales $(91,5 \%)$, principalmente los relacionados con el vulcanismo. Esto puede deberse también a la presencia en la Escuela de diversos carteles, maquetas y películas referentes a este tema o a la imagen que se suele ofrecer de este tema desde la comunicación social. Es importante realzar el bajo porcentaje de respuestas $(2,3 \%)$ referentes a las competencias desarrolladas. Este porcentaje puede denunciar la importancia dada por los alumnos a los contenidos conceptuales en detrimento del desarrollo de competencias, en concordancia con la importancia atribuida a los exámenes finales. Nótese, sin embargo, que la evaluación en Ciencias Naturales tuvo 
siempre en cuenta ambos criterios, habiendo asumido un papel preponderante el desarrollo de competencias.

\begin{tabular}{|c|c|c|c|c|c|}
\hline & & & Categoría de re & puestas & \\
\hline & Categoría & Número de & & bcategoría & Número de \\
\hline & & & & 1.1.1- No especifica & $54(31,4 \%)$ \\
\hline & & & & $\begin{array}{l}\text { 1.1.2- Estructura del } \\
\text { volcán }\end{array}$ & $12(7,0 \%)$ \\
\hline & & & & $\begin{array}{l}\text { 1.1.3- Materiales } \\
\text { expelidos }\end{array}$ & $7(4,1 \%)$ \\
\hline & & & & 1.1.4- Tipos de erupción & $6(3,5 \%)$ \\
\hline & & & $\begin{array}{l}\text { 1.1-Volcanes } \\
(58.3 \%)\end{array}$ & $\begin{array}{l}\text { 1.1.5- Funcionamiento de } \\
\text { los volcanes }\end{array}$ & $7(4,1 \%)$ \\
\hline த் & & & & $\begin{array}{l}\text { 1.1.6- Consecuencias de } \\
\text { un volcán }\end{array}$ & $9(5,2 \%)$ \\
\hline 5 & & & & $\begin{array}{l}\text { 1.1.7- Actitud a adoptarse } \\
\text { en caso de erupción }\end{array}$ & $1(0,6 \%)$ \\
\hline एँ & & & & $\begin{array}{l}\text { 1.1.8- Localización de los } \\
\text { volcanes }\end{array}$ & $3(1,8 \%)$ \\
\hline$\frac{\bar{a}}{\pi}$ & 1-Contenidos & $157(91,3 \%)$ & & 1.1.9- Tipo de volcán & $1(0,6 \%)$ \\
\hline ڤ & & & & 1.2.1- No especifica & $9(5,2 \%)$ \\
\hline$\stackrel{0}{2}$ & & & & 1.2.2- Enuncia conceptos & $19(11,0 \%)$ \\
\hline ני & & & & $\begin{array}{l}\text { 1.2.3- Causas de los } \\
\text { sismos }\end{array}$ & $5(2,9 \%)$ \\
\hline & & & $(23,2 \%)$ & 1.2.4- Tipos de sismos & $1(0,6 \%)$ \\
\hline & & & & 1.2.5- Sismógrafos & $4(2,3 \%)$ \\
\hline & & & & \begin{tabular}{|l|} 
1.2.6- Escalas de \\
clasificación de sismos
\end{tabular} & $2(1,2 \%)$ \\
\hline & & & $\begin{array}{l}\text { 1.3- Estructura de } \\
\text { la Tierra } \\
(2,3 \%)\end{array}$ & & $4(2,3 \%)$ \\
\hline & & & $\begin{array}{c}\text { 1.4- No especifica } \\
(7,5 \%)\end{array}$ & & $13(7,5 \%)$ \\
\hline & $\begin{array}{l}2 \text { - Competencias } \\
\text { desarrolladas }\end{array}$ & $4(2,3 \%)$ & & & $4(2,3 \%)$ \\
\hline & 3- No responde & $7(4,1 \%)$ & & & $7(4,1 \%)$ \\
\hline & $\begin{array}{l}\text { 4- Respuesta no } \\
\text { válida }\end{array}$ & $4(2,3 \%)$ & & & $4(2,3 \%)$ \\
\hline & & & Total de respuesta & & 172 \\
\hline
\end{tabular}

Cuadro 3.- Respuestas de los alumnos en la dimensión C.

En la dimensión D, "Concienciación de lo aprendido", el Cuadro 4 resume el análisis de las respuestas de los alumnos.

Fueron muchas las respuestas incluidas (27) en la categoría 8. Interpretamos que los alumnos no comprendieron la pregunta, confundiendo "Concienciación de lo aprendido" con "tarea realizada". Aunque este análisis no revele el número de respuestas incluidas en esta categoría, disminuye a medida que transcurrían las clases 
y, consecuentemente, la aplicación de este instrumento, ya que la profesora aclaró esa ambigüedad inmediatamente que se dio cuenta de la dificultad.

\begin{tabular}{|c|c|c|}
\hline \multirow{10}{*}{ 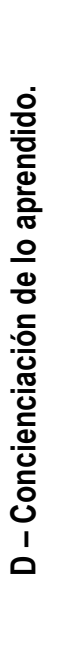 } & \multicolumn{2}{|c|}{ Categoría de respuestas } \\
\hline & Categoría & $\begin{array}{c}\text { Número de } \\
\text { respuestas } \\
(\%)\end{array}$ \\
\hline & 1- Actitud del alumno (Atención; empeño, participación) & $17(12,1 \%)$ \\
\hline & 2- Buenos resultados de evaluación sumativa & $4(2,8 \%)$ \\
\hline & 3- Comprensión de los contenidos conceptuales & $20(14,2 \%)$ \\
\hline & 4- Enunciado de contenidos conceptuales & $7(5,0 \%)$ \\
\hline & 5- Realización de actividades (ejercicios, trabajos) & $32(22,7 \%)$ \\
\hline & 6- Memorización de los contenidos conceptuales & $3(2,1 \%)$ \\
\hline & 7- No responde & $31(22,0 \%)$ \\
\hline & 8- Respuesta no válida & $27(19,1 \%)$ \\
\hline & Total de respuesta & 141 \\
\hline
\end{tabular}

Cuadro 4.- Respuestas de los alumnos en la dimensión D.

Las respuestas de los alumnos en la dimensión E, "Formulación de dudas" (E), fueron también motivo de análisis (Cuadro 5), verificándose que la mayor parte de los alumnos (82 respuestas) no formula preguntas.

\begin{tabular}{|c|c|c|c|c|}
\hline \multirow{9}{*}{ 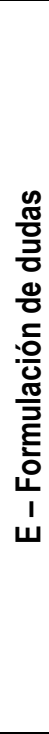 } & \multicolumn{4}{|c|}{ Categoría de respuestas } \\
\hline & Categoría & $\begin{array}{c}\text { Número de } \\
\text { respuestas } \\
(\%)\end{array}$ & Subcategoría & $\begin{array}{c}\text { Número de } \\
\text { respuestas } \\
(\%)\end{array}$ \\
\hline & \multirow{3}{*}{ 1- Contenidos conceptuales } & \multirow{3}{*}{$23(19,3 \%)$} & 1.1-Volcanes & $18(15,1 \%)$ \\
\hline & & & 1.2- Sismos & $4(3,4 \%)$ \\
\hline & & & $\begin{array}{l}\text { 1.3- Relación entre } \\
\text { volcanes y sismos }\end{array}$ & $1(0,8 \%)$ \\
\hline & $\begin{array}{l}\text { 2- Planificación de actividades a } \\
\text { dinamizar }\end{array}$ & $10(8,4 \%)$ & & $10(8,4 \%)$ \\
\hline & $\begin{array}{l}\text { 3- Actitud de los alumnos } \\
\text { (Atención y/o empeño) }\end{array}$ & $2(1,7 \%)$ & & $2(1,7 \%)$ \\
\hline & 4- No responde & $82(68,9 \%)$ & & $82(68,9 \%)$ \\
\hline & 5- Respuesta no válida & $2(1,7 \%)$ & & $2(1,7 \%)$ \\
\hline & & & Total de respuestas & 119 \\
\hline
\end{tabular}

Cuadro 5.- Respuestas de los alumnos en la dimensión E.

El elevado porcentaje de alumnos que no expone ninguna duda $(68,9 \%)$ puede reflejar: i) la dificultad que los alumnos sienten a la hora de preguntar (de hecho, durante la trayectoria educativa de los alumnos, éstos habitualmente no preguntan, 
aunque responden a las preguntas de los profesores); ii) una cierta vergüenza y/o recelo asociado al planteamiento de dudas y; iii) a la inexistencia de dudas.

\section{Análisis e interpretación de los Diarios de Clase de los alumnos}

En el análisis de los datos contenidos en los Diarios de Clase, se llevó a cabo la metodología de análisis e interpretación de los Diarios de Clase de los profesores, definida por Zabalza (1994). Esta metodología se refiere a dos fases: 1) primera lectura exploratoria y 2) segunda lectura acompañada por anotaciones al margen, selección de informaciones y datos relevantes en relación a las áreas temáticas importantes para la obtención de los objetivos del estudio.

Se procedió así a la clasificación de los Diarios de Clase y al análisis de las tareas realizadas.

Éstos son diarios de características mixtas ya que surgen descripciones a veces con algún pormenor: i) de las tareas realizadas por profesores y alumnos en el aula - "La clase empezó con la corrección del trabajo de casa (TDC). Inicialmente pregunté quién no había hecho el TDC. Todos los alumnos dijeron haberlo hecho" (Diario de Clase $1)$; ii) de las características de los alumnos y del modo como el profesor actúa y se siente - "(...), aunque a veces siento que se decepcionan, lo que a veces también influye en mí." (Diario de Clase - 2). En general, fue difícil distinguir algunas rutinas del aula, atendiendo al número reducido (6) de diarios de clase realizados. No obstante, es posible identificar las dos rutinas siguientes: i) la solicitud de participación en la aclaración de algunos términos a partir del conocimiento previo de los alumnos o de la interpretación de una figura, teniendo en cuenta su definición o explicación ("(...) pedí para que los alumnos interpretasen las figuras referentes a la formación de una caldera presentadas en la transparencia y que me explicasen de qué se trataba." (Diario de Clase - 1); "Seguidamente inicié el debate sobre los términos: sismo, sismología, terremoto y "tsunami" o maremoto. En este debate traté de que los alumnos expusieran sus ideas sobre estos términos." (Diario de Clase - 4); e ii) la corrección de las fichas de trabajo realizadas en casa o durante la clase en la pizarra con la colaboración activa de los alumnos, consistiendo primero en la respuesta oral y después ir a la pizarra para escribirla, lo más completa posible ("Todas las respuestas fueron escritas en la pizarra por uno de los alumnos que respondió oralmente." (Diario de Clase - 1); "Las respuestas fueron escritas por dos alumnos en la pizarra." (Diario de Clase - 4).

El análisis individual de los diarios, como ya se ha referido, incidió solamente en las tareas y en los dilemas. El cuadro 6 representa los aspectos de la tarea relacionados con: la descripción de las actividades propuestas por el profesor; la finalidad de la actividad propuesta y la descripción del desempeño del alumno y de los recursos.

Este cuadro nos muestra que la profesora verbalizó, durante los Diarios de Clase, la finalidad de cada actividad, así como la descripción de la actividad y del desempeño de los alumnos en esas actividades. Es también relevante la variedad de recursos utilizados por la profesora, lo cual fue destacado como positivo por los alumnos en los Snapshots. 
S. CARRASQUINHO, C. VASCONCELOS, N. COSTA

\begin{tabular}{|c|c|c|c|c|}
\hline \multirow{2}{*}{$\begin{array}{l}\mathrm{N}^{0} \text { de } \\
\text { diario }\end{array}$} & \multicolumn{4}{|c|}{ Estructura de la tarea } \\
\hline & Objetivo de la actividad propuesta & $\begin{array}{l}\text { Descripción de las actividades } \\
\text { propuestas por el profesor }\end{array}$ & $\begin{array}{l}\text { Descripción del } \\
\text { desempeño del } \\
\text { alumno }\end{array}$ & Recursos \\
\hline 1 & $\begin{array}{l}\text { Motivar a los alumnos para que realicen } \\
\text { la corrección del trabajo de casa. } \\
\text { Iniciar la pregunta - problema que } \\
\text { servirá de base a la realización de la } 1^{\text {a }} \\
\text { parte de la planificación. } \\
\text { Aclarar los conocimientos que los } \\
\text { alumnos poseen, aclarar dudas } \\
\text { emergentes de la Ficha diagnóstico y } \\
\text { conceptualizar los conceptos previstos } \\
\text { en la planificación. } \\
\text { Verificar la comprensión de los } \\
\text { conceptos por parte de los alumnos, así } \\
\text { como resumir las ideas debatidas. }\end{array}$ & $\begin{array}{l}\text { La profesora anota el número de } \\
\text { alumnos que no realizaron el } \\
\text { trabajo de casa e interviene en } \\
\text { su corrección. } \\
\text { Orientación en la formulación del } \\
\text { problema. } \\
\text { Mediación en la exploración de la } \\
\text { presentación en PowerPoint y en } \\
\text { la transparencia no } 1 \text {, solicitando } \\
\text { la participación de los alumnos } \\
\text { en el análisis e interpretación de } \\
\text { las diversas imágenes. } \\
\text { Promoción de la síntesis de los } \\
\text { conceptos debatidos durante la } \\
\text { actividad anterior en la pizarra } \\
\text { blanca. }\end{array}$ & $\begin{array}{l}\text { Participación en la } \\
\text { corrección del trabajo } \\
\text { de casa. } \\
\text { Formulación del } \\
\text { problema. } \\
\text { Interpretación y } \\
\text { conceptualización de } \\
\text { los diferentes } \\
\text { contenidos. } \\
\text { Colaboración activa } \\
\text { en la síntesis de los } \\
\text { contenidos. }\end{array}$ & $\begin{array}{l}\text { Pizarra } \\
\text { Pizarra } \\
\text { Presentación en } \\
\text { PowerPoint, } \\
\text { Computador, } \\
\text { Proyector multimedia, } \\
\text { Transparencia n }{ }^{0} 1, \\
\text { Retroproyector, } \\
\text { Pizarra }\end{array}$ \\
\hline 2 & $\begin{array}{l}\text { Demostrar a los alumnos la existencia } \\
\text { de actividad volcánica además de } \\
\text { aquella existente en las Azores. Por } \\
\text { otro lado, esta película atribuye } \\
\text { bastante importancia al papel } \\
\text { desempeñado por los científicos que } \\
\text { estudian los volcanes, factor que se } \\
\text { consideró pertinente atendiendo al alto } \\
\text { porcentaje de alumnos que, de acuerdo } \\
\text { con la ficha diagnóstico, no conoce ni } \\
\text { siquiera la designación atribuida a } \\
\text { estos científicos. } \\
\text { Abordar conceptos relativos al } \\
\text { vulcanismo secundario, con el fin de } \\
\text { aclarar las dudas de los alumnos en los } \\
\text { Snapshots. }\end{array}$ & $\begin{array}{l}\text { Facilitar la visualización de una } \\
\text { película, seguida de la } \\
\text { Resolución de la ficha } n^{0} 1, \\
\text { referente a conceptos abordados } \\
\text { en la película. Después continúa } \\
\text { la mediación de la corrección, el } \\
\text { análisis de la ficha y de algunos } \\
\text { conceptos con apoyo del manual } \\
\text { adoptado. }\end{array}$ & $\begin{array}{l}\text { Visualización de la } \\
\text { película para resolver } \\
\text { la ficha de trabajo } \\
\text { subsiguiente. }\end{array}$ & $\begin{array}{l}\text { Película, } \\
\text { Vídeo, } \\
\text { Fotocopias de la ficha } \\
\text { de trabajo } n^{0} 1 \text {, } \\
\text { Manual EureKa }\end{array}$ \\
\hline 3 & $\begin{array}{l}\text { Aclarar dudas sobre los contenidos } \\
\text { abordados. } \\
\text { Formar grupos de trabajo según las } \\
\text { reglas definidas y teniendo también en } \\
\text { cuenta lo que ellos elijan. }\end{array}$ & $\begin{array}{l}\text { Aclaración de dudas planteadas } \\
\text { por los alumnos en los } \\
\text { Snapshots. } \\
\text { Mediación en la formación de } \\
\text { grupos de trabajo y en el trabajo } \\
\text { de grupo, con el objetivo de } \\
\text { intentar resolver la pregunta } \\
\text { central, así como otras que } \\
\text { vayan surgiendo. }\end{array}$ & $\begin{array}{l}\text { Planteamiento de } \\
\text { dudas } \\
\text { Colaboración en la } \\
\text { formación de los } \\
\text { grupos }\end{array}$ & \\
\hline 4 & $\begin{array}{l}\text { Desarrollar la autonomía y } \\
\text { competencias inherentes al trabajo de } \\
\text { grupo: cooperación y respeto a la } \\
\text { divergencia. }\end{array}$ & $\begin{array}{l}\text { Mediación en el trabajo de } \\
\text { investigación de los diferentes } \\
\text { grupos en la biblioteca. }\end{array}$ & $\begin{array}{l}\text { Investigación y } \\
\text { elaboración de } \\
\text { trabajos sobre el } \\
\text { tema. }\end{array}$ & $\begin{array}{l}\text { Computadores, Sitios } \\
\text { en Internet, } \\
\text { Libros, } \\
\text { Publicaciones }\end{array}$ \\
\hline
\end{tabular}

Cuadro 6.- Aspectos relacionados con la tarea identificados en los Diarios de Clase. 


\begin{tabular}{|c|c|c|c|c|}
\hline \multirow{2}{*}{$\begin{array}{l}\mathrm{N}^{0} \text { de } \\
\text { diario }\end{array}$} & \multicolumn{4}{|c|}{ Estructura de la tarea } \\
\hline & Objetivo de la actividad propuesta & $\begin{array}{l}\text { Descripción de las actividades } \\
\text { propuestas por el profesor }\end{array}$ & $\begin{array}{l}\text { Descripción del } \\
\text { desempeño del } \\
\text { alumno }\end{array}$ & Recursos \\
\hline 5 & $\begin{array}{l}\text { Concienciar a los alumnos sobre la } \\
\text { importancia del cumplimiento de } \\
\text { plazos. } \\
\text { Iniciar la pregunta - problema que } \\
\text { servirá de base a la realización de la } 2^{\text {a }} \\
\text { parte de la planificación. } \\
\text { Aclarar los conocimientos que los } \\
\text { alumnos poseen, aclarar dudas } \\
\text { surgidas de la ficha diagnostico y } \\
\text { conceptualización de los conceptos } \\
\text { previstos en la planificación. } \\
\text { Proporcionar que los alumnos } \\
\text { respondan a parte de la pregunta - } \\
\text { problema. } \\
\text { Resolución y colaboración en la } \\
\text { corrección de la ficha de trabajo. }\end{array}$ & $\begin{array}{l}\text { Orientación en la formulación del } \\
\text { problema. } \\
\text { Fomentar el debate sobre } \\
\text { diversos conceptos relacionados } \\
\text { con los Sismos y la respectiva } \\
\text { síntesis. } \\
\text { Mediación en la Resolución y } \\
\text { corrección de una ficha de } \\
\text { trabajo }\end{array}$ & $\begin{array}{l}\text { Entrega de los } \\
\text { trabajos de grupo. } \\
\text { Formulación del } \\
\text { problema. } \\
\text { Debate de ideas y } \\
\text { síntesis sobre los } \\
\text { conceptos propuestos } \\
\text { por la profesora }\end{array}$ & $\begin{array}{l}\text { Fotocopias de la ficha } \\
\text { de trabajo } n^{0} 2 \\
\text { Pizarra }\end{array}$ \\
\hline 6 & $\begin{array}{l}\text { Explicación del modo de } \\
\text { funcionamiento de los sismógrafos } \\
\text { Interiorización del concepto de isosista. } \\
\text { Concienciación sobre la existencia de } \\
\text { diferentes modelos referentes al interior } \\
\text { de la tierra. } \\
\text { Revisión de los conceptos abordados. }\end{array}$ & $\begin{array}{l}\text { Mediación en la interpretación de } \\
\text { la figura } 1 \text { de la transparencia } n^{0} \\
2 . \\
\text { Mediación en la resolución y } \\
\text { corrección de una ficha de } \\
\text { trabajo. } \\
\text { Mediación en la interpretación de } \\
\text { las figuras de sismogramas y } \\
\text { sismógrafos de la transparencia } \\
n^{0} 2 \text {. } \\
\text { Mediación en la Resolución y } \\
\text { corrección de una ficha de } \\
\text { trabajo de las páginas } 23 \text { y } 24 \\
\text { del manual escolar Eureka. } \\
\text { Mediación en la interpretación de } \\
\text { las figuras de la transparencia } n^{0} \\
3 \text {. } \\
\text { Fomentar la revisión de los } \\
\text { conceptos debatidos. }\end{array}$ & $\begin{array}{l}\text { Interpretación de la } \\
\text { figura. } \\
\text { Resolución y } \\
\text { colaboración en la } \\
\text { corrección de la ficha } \\
\text { de trabajo. } \\
\text { Interpretación de las } \\
\text { figuras. } \\
\text { Resolución y } \\
\text { colaboración en la } \\
\text { corrección de la ficha } \\
\text { de trabajo. } \\
\text { Interpretación de las } \\
\text { figuras. } \\
\text { Colaboración activa } \\
\text { en la revisión de los } \\
\text { contenidos }\end{array}$ & $\begin{array}{l}\text { Manual escolar } \\
\text { "Eureka - tierra en } \\
\text { transformación II" } \\
\text { Transparencia n } 3 \\
\text { Retroproyector } \\
\text { Mapas de Conceptos }\end{array}$ \\
\hline
\end{tabular}

Cuadro 6 (continuación).- Aspectos relacionados con la tarea identificados en los Diarios de Clase.

Los dilemas son analizados de acuerdo con lo que defiende Zabalza (1994). A continuación se describe en el cuadro 7 el análisis de los dilemas con los que la profesora se encontró. 


\begin{tabular}{|c|c|c|}
\hline $\begin{array}{l}\mathrm{N}^{0} \text { de } \\
\text { diario }\end{array}$ & Dilema & Resolución \\
\hline \multirow[b]{2}{*}{1} & $\begin{array}{l}\text { Valoración de la participación versus Indisciplina } \\
\text { La profesora expresa frecuentemente su deseo de que los alumnos participen activamente en el } \\
\text { debate de los diversos temas, no obstante se encuentra con una participación desordenada y confusa. } \\
\text { De este modo, la profesora se debate entre la importancia de la participación de todos los alumnos y } \\
\text { de preguntas de orden disciplinar que faciliten la concentración y la comprensión del mensaje. } \\
\text { "Los alumnos demostraron, como ya se ha hecho costumbre, bastante interés en participar en la } \\
\text { corrección del TPC, participando y esforzándose. A veces, el deseo de participar y mostrar su trabajo } \\
\text { era tanto, que respondían simultáneamente sin levantar el brazo o también respondian levantando el } \\
\text { brazo y hablando al mismo tiempo, sin esperar mi autorización, lo que impedía que ellos mismos se } \\
\text { escuchasen." } \\
\text { "Nuevamente aquí surgió cierta confusión, todos querían hablar" } \\
\text { Este dilema surgirá igualmente en otras situaciones de debate en grupo aula, no obstante su } \\
\text { importancia va disminuyendo atendiendo a una mejor actitud de los alumnos en esta situación en la } \\
\text { clase. }\end{array}$ & $\begin{array}{l}\text { Para resolver este dilema la profesora llamó } \\
\text { la atención a los alumnos, explicando las } \\
\text { ventajas de no hablar al unísono o de } \\
\text { esperar el turno de cada cual. } \\
\text { "Estas situaciones originan un poco de } \\
\text { desorden, por lo que para resolverla acudí } \\
\text { varias veces a frases como "no les oigo si } \\
\text { hablan al mismo tiempo" o "si interrumpen al } \\
\text { compañero, ni le oigo a él ni a ustedes." }\end{array}$ \\
\hline & $\begin{array}{l}\text { Autonomía versus Dependencia } \\
\text { El dilema que confronta la autonomía versus la dependencia de los alumnos va a surgir en varias } \\
\text { clases, aunque estará subordinado a diferentes situaciones. Durante esta actividad se constata que la } \\
\text { profesora desea fomentar la autonomía de decisión en los alumnos. } \\
\text { "Sugerí que los alumnos decidiesen si preferían copiar o no en el cuaderno lo que estaba escrito en la } \\
\text { pizarra." } \\
\text { Sin embargo ella no consigue desprenderse de la importancia que escribir los contenidos en la libreta } \\
\text { tiene para ella, por ello enumera las ventajas de ejecutar esa tarea, acabando por influenciar a los } \\
\text { alumnos. } \\
\text { "Frente a la incapacidad de los alumnos para decidir, me pregunté si debía obligarlos a copiar, o sea, a } \\
\text { decidir por ellos." } \\
\text { "(...) decidí insistir en las ventajas de copiar, pero también mencioné donde podían consultar esta } \\
\text { información y conceptos de la clase en el manual Escolar" }\end{array}$ & $\begin{array}{l}\text { La profesora contribuyó a que los alumnos } \\
\text { dependiesen de su decisión, sin haber sido } \\
\text { estimulados a ponderar los pros y contras de } \\
\text { esta opción, tarea que en su día a día se } \\
\text { asumirá como fundamental para el ejercicio } \\
\text { de una ciudadanía activa. } \\
\text { "Confío en que la mayoría de los alumnos } \\
\text { copió, sin embargo pienso que sólo fue así } \\
\text { ya que pensaron que me agradaría que lo } \\
\text { hiciesen, y tal vez, inconscientemente, yo } \\
\text { haya transmitido que esa opción sería la } \\
\text { más apreciada por mí." } \\
\text { La profesora no consiguió resolver este } \\
\text { conflicto, ya que en el Diario reconoció que } \\
\text { transmitió involuntariamente su juicio de } \\
\text { valores sobre esa opción, lo que, } \\
\text { inconscientemente, pudo haber influido en la } \\
\text { decisión de los alumnos, retirándoles la } \\
\text { posibilidad de ejercer su autonomía. }\end{array}$ \\
\hline \multirow[t]{2}{*}{2} & $\begin{array}{l}\text { Estrategia motivadora versus Estrategia lúdica } \\
\text { La profesora reflexiona sobre el objetivo inherente a la visualización de una película. Aunque la } \\
\text { profesora muestre preocupación en relacionar el contenido conceptual con situaciones reales, ella } \\
\text { reflexiona sobre la eficacia y utilidad de este tipo de estrategia. Este dilema parece tener su génesis en } \\
\text { la concepción que los alumnos tienen sobre la visualización de películas. } \\
\text { "Realmente, a veces me quedo con la duda de si este tipo de metodología será eficaz con este nivel de } \\
\text { edades. Tiene varias ventajas, como por ejemplo la de establecer una conexión con situaciones reales } \\
\text { y dramáticas, y proporcionar una dinámica de clase diferente, sin embargo me pregunto si esta } \\
\text { metodología será realmente útil para estos alumnos, ya que ellos tomaron esta actividad como un } \\
\text { momento lúdico.(...) Frente a esta información, los alumnos se sorprendieron y tuvieron incluso miedo } \\
\text { de la evaluación, por lo que me di cuenta que ellos asociaban la visualización de una película a } \\
\text { objetivos de motivación o de "pasar el tiempo" y no como un recurso pasible de ser eficaz para el } \\
\text { proceso de enseñanza y aprendizaje. Sin embargo, cuando mencione la palabra "ficha" pensaron que } \\
\text { iría a evaluar la atención que prestarían a la película." }\end{array}$ & $\begin{array}{l}\text { Para solucionar este dilema la profesora } \\
\text { destacó la importancia de la ficha relativa a } \\
\text { la película. } \\
\text { "En este momento, les informé que } \\
\text { tendrían que resolver una ficha sobre los } \\
\text { conceptos abordados en el filme, } \\
\text { destacando que no se trataría de un } \\
\text { momento lúdico." }\end{array}$ \\
\hline & $\begin{array}{l}\text { Autonomía versus Dependencia } \\
\text { La profesora vuelve a confrontarse con este dilema en la resolución de la ficha que se refiere a los } \\
\text { conceptos abordados en la película. Aunque la profesora muestre que quiere fomentar la autonomía de } \\
\text { los alumnos, ella se siente influenciada por la decepción de los alumnos en relación a su tentativa de } \\
\text { ayudarlos a ser autónomos. De esta manera, aunque exprese por escrito el deseo de promover la } \\
\text { autonomía, la influencia que la decepción de los alumnos ejerce sobre la profesora puede evidenciar } \\
\text { algunas dudas entre el desarrollo de esta competencia y la desmotivación que puede resultar de la } \\
\text { decepción de los alumnos frente a la ausencia del refuerzo positivo inmediato. } \\
\text { "durante la resolución estuve siempre dispuesta a aclarar dudas pero me di cuenta que en la mayoría } \\
\text { de los casos, los alumnos me llamaban para confirmar la respuesta. En general, a ese tipo de dudas } \\
\text { respondo que la corrección se hará en conjunto con todo el grupo, para que podamos debatir las } \\
\text { diferentes opiniones, (deseo de la profesora en promover la autonomía) aunque a veces siento que se } \\
\text { decepcionan, lo que en ocasiones también acaba influyendo en mí." }\end{array}$ & $\begin{array}{l}\text { Relativamente a este dilema, la profesora } \\
\text { opta por promover la autonomía de los } \\
\text { alumnos, enfrentando la decepción de éstos. }\end{array}$ \\
\hline
\end{tabular}

Cuadro 7.- Análisis de los dilemas identificados en los Diarios de Clase y sus soluciones. 


\begin{tabular}{|c|c|c|}
\hline $\begin{array}{l}\mathrm{N}^{0} \text { de } \\
\text { diario }\end{array}$ & Dilema & Resolución \\
\hline \multirow{3}{*}{3} & $\begin{array}{l}\text { Grupo versus Individuo } \\
\text { Este dilema se refleja en la duda planteada por la profesora sobre la efectividad del trabajo de grupo. } \\
\text { "A veces yo misma tengo dudas sobre la efectividad del trabajo en grupo ya que no es tan eficaz el } \\
\text { apoyo que he dado a los alumnos con mayores dificultades" } \\
\text { La docente se pregunta sobre la ventaja de la colaboración en grupo, atendiendo a que el apoyo a los } \\
\text { alumnos con más dificultades deja de ser permanente. Por otro lado, esos alumnos presentan } \\
\text { dificultades para relacionarse con los otros alumnos ya que plantean que los otros alumnos no valoran } \\
\text { el trabajo de éstos y por eso no quieren trabajar en grupo. } \\
\text { "La mayoría de los alumnos con mayores dificultades se manifestaron en contra, refiriendo que este } \\
\text { tipo de trabajo les perjudica ya que los mejores alumnos quedan siempre juntos." } \\
\text { En vez de fomentar la cooperación, una de las interrogantes que la profesora tiene que resolver es la } \\
\text { posibilidad de que este tipo de trabajo pueda contribuir a la exclusión de los alumnos con más } \\
\text { dificultades y consecuente frustración. Los dilemas Cooperación versus Exclusión entre los alumnos } \\
\text { "buenos" y los alumnos con más dificultades, y Frustración versus Empeño de los alumnos "buenos" y } \\
\text { de los alumnos con más dificultades se entremezclan en este dilema. Este último dilema se refiere a la } \\
\text { frustración de los alumnos con más dificultades, ya que sienten que los restantes alumnos no valoran } \\
\text { sus trabajos, lo que puede originar un sentido de desmotivación y la frustración que los "buenos" } \\
\text { alumnos pueden sentir en relación al trabajo de los alumnos con más dificultades. } \\
\text { "La formación de grupos es una tarea siempre difíil, en la cual me encuentro con varias } \\
\text { ambigüedades: i) hacer los grupos yo sola, imponiéndolos, o de acuerdo con los alumnos, negociando. } \\
\text { ii) Evitar grupos únicamente de "buenos alumnos" o solamente de alumnos con más dificultades, o } \\
\text { dinamizar la cooperación intergrupo entre alumnos de diferentes niveles y enfrentarme a la frustración } \\
\text { de los alumnos con más interés y empeño." }\end{array}$ & $\begin{array}{l}\text { Para intentar solucionar el dilema Grupo } \\
\text { versus Individuo, la profesora usó una } \\
\text { estrategia de formación de grupos } \\
\text { atendiendo a diversos criterios. } \\
\text { "Frente a la primera duda, utilicé una } \\
\text { estrategia múltipla, permitiéndoles elegir el } \\
\text { grupo pero colaborando activamente en esa } \\
\text { discusión, para cumplir las reglas dispuestas } \\
\text { desde el inicio, certificándome siempre que } \\
\text { aunque no fuese la primera opción de } \\
\text { algunos, ellos concordarían en trabajar con } \\
\text { ese grupo. Siento siempre alguna dificultad } \\
\text { en imponer el trabajo de grupo,(...)" }\end{array}$ \\
\hline & $\begin{array}{l}\text { Imposición versus Negociación } \\
\text { Este dilema está inherente a todo el proceso de formación de los grupos de trabajo. La profesora se } \\
\text { debate entre los criterios que cree son importantes para una correcta realización del trabajo de grupo y } \\
\text { aquellos que los alumnos eligen. } \\
\text { "Sin embargo la formación de los grupos fue sometida a dos condiciones que ya yo había pensado } \\
\text { previamente: i) serán en total } 5 \text { grupos, } 4 \text { de } 5 \text { elementos y } 1 \text { de 4; ii) todos los grupos tienen que estar } \\
\text { constituidos por } 2 \text { varones y } 2 \text { hembras (exceptuando un grupo, ya que el número de chicos no era } \\
\text { suficiente (uno de los grupos se quedó solamente con chicas). La formación de grupos es una tarea } \\
\text { siempre dificil, en la cual me enfrento a varias indecisiones: i) hacer los grupos yo sola, imponiéndolos, } \\
\text { o en acuerdo con los alumnos, negociando (...)" }\end{array}$ & $\begin{array}{l}\text { La resolución del dilema anterior permitió } \\
\text { también la resolución de este dilema. }\end{array}$ \\
\hline & $\begin{array}{l}\text { Autonomía versus Dependencia } \\
\text { Durante la obtención de información, el dilema Autonomía versus Dependencia incide nuevamente en } \\
\text { la descripción de la profesora. } \\
\text { "(...) intenté orientarlos para que se volvieran más autónomos, sin embargo verifico que todos los } \\
\text { grupos aún necesitan bastante refuerzo positivo y apoyo, lo que hace difíil la orientación del trabajo } \\
\text { de grupo." }\end{array}$ & $\begin{array}{l}\text { Para intentar resolver este conflicto, la la } \\
\text { profesora recurrió al sentido de cooperación } \\
\text { y ayuda de los alumnos que mejor dominan } \\
\text { los medios de investigación, consiguiendo } \\
\text { sentir que esta resolución resolvió también } \\
\text { el dilema de la Frustración versus Empeño. } \\
\text { "Frente a esta situación pedí que los } \\
\text { alumnos que mejor conocen el modo de } \\
\text { funcionamiento de Internet cooperasen con } \\
\text { los restantes, incluso de grupos diferentes, y } \\
\text { que también colaborasen en la búsqueda de } \\
\text { información bibliográfica. Verifiqué que de } \\
\text { este modo conseguí también resolver mi } \\
\text { tercera duda y superar la posible frustración } \\
\text { de los "buenos" alumnos, ya que ellos } \\
\text { estaban satisfechos con su papel de } \\
\text { "monitores", lo que vino a resolver mi } \\
\text { segunda duda (dilema Frustración versus } \\
\text { Empeño)." }\end{array}$ \\
\hline 4 & $\begin{array}{l}\text { Flexibilidad versus Rigidez } \\
\text { Este dilema se refiere al ambiente creado por la entrega de los trabajos. La profesora oscila entre la } \\
\text { posibilidad de alargar el plazo de entrega o la de ser inflexible, no recibiendo trabajos fuera del plazo } \\
\text { estipulado. Esta duda parece surgir, por un lado, debido a que la profesora considera importante la } \\
\text { constatación, por parte de los alumnos, de la importancia de cualquier plazo de entrega y de las } \\
\text { penalizaciones inherentes a su incumplimiento, y por otro, ser sensible a la preocupación que los } \\
\text { alumnos mostraron, ya que se trata de un elemento de evaluación sumativa. } \\
\text { "Algunos alumnos argumentaron no haber acabado el trabajo y me pidieron que prolongara la hecha } \\
\text { de entrega. En este tipo de situaciones, en relación a la perturbación y preocupación demostrada por } \\
\text { los alumnos, debido a que se trata de un elemento de evaluación, siempre tengo dudas sobre si debo } \\
\text { hacer lo que me piden o si debo adoptar una actitud inflexible, ya que las fechas de entrega de los } \\
\text { trabajos son esenciales en cualquier área de nuestra sociedad." }\end{array}$ & $\begin{array}{l}\text { Para resolver este dilema la profesora acude } \\
\text { nuevamente a una estrategia intermedia. } \\
\text { "Posteriormente, decidí que podian entregar } \\
\text { los trabajos en la próxima clase, pero que no } \\
\text { obstante perderian los puntos atribuidos al } \\
\text { criterio de evaluación "Entrega en el plazo } \\
\text { definido" }\end{array}$ \\
\hline
\end{tabular}

Cuadro 7 (continuación).- Análisis de los dilemas identificados en los Diarios de Clase y sus soluciones. 


\begin{tabular}{|c|c|c|}
\hline 5 & $\begin{array}{l}\text { Cumplimiento del programa versus Interés (expectativas) de los alumnos } \\
\text { Esta modificación de la planificación le provocó un dilema a la profesora, ya que ella siente que, } \\
\text { por un lado, al retirar la posibilidad de realizar este trabajo defrauda las expectativas de los } \\
\text { alumnos de resolver parte de la pregunta-problema autónomamente; por otro lado es esencial } \\
\text { asegurar que el nivel conceptual de estos alumnos sea idéntico al de los restantes grupos de la } \\
\text { escuela. } \\
\text { "(...)Esta alteración me produjo algunas dudas ya que los alumnos se empeñaron bastante en el } \\
\text { trabajo anterior y deseaban descubrir por ellos mismos la respuesta a muchas de sus dudas." }\end{array}$ & $\begin{array}{l}\text { En este dilema la profesora optó por el } \\
\text { cumplimiento del programa, ya que } \\
\text { consideró que éste contribuye para la } \\
\text { igualdad de oportunidades en el próximo } \\
\text { año lectivo. } \\
\text { "Sin embargo, influyó en esta decisión el } \\
\text { hecho de que en términos conceptuales } \\
\text { los alumnos puedan llegar el mismo nivel } \\
\text { de los otros alumnos de la escuela." }\end{array}$ \\
\hline 6 & $\begin{array}{l}\text { Autonomía versus Dependencia } \\
\text { En la respuesta a la última pregunta los alumnos volvieron a demostrar una relativa dependencia } \\
\text { del refuerzo positivo de la profesora. } \\
\text { "En esta pregunta, intentaron llamarme para que les dijera la respuesta, revelando dependencia. } \\
\text { Pienso que esto se debió a que se trataba de una pregunta que implicaba cierta reflexión y no } \\
\text { solamente una interpretación de figuras o respuesta directa. De esta forma, verifiqué que no } \\
\text { obstante a ellos ser más autónomos, evidencian aún alguna dificultad siempre que las preguntas } \\
\text { encierran raciocinios más complejos." }\end{array}$ & $\begin{array}{l}\text { En esta clase la profesora resolvió este } \\
\text { dilema optando por la autonomía, ya que } \\
\text { se trataba del fin del año escolar. } \\
\text { "No obstante, traté que escribiesen } \\
\text { únicamente lo que pensaban, pues } \\
\text { estamos en el fin del año escolar y } \\
\text { consecuentemente ya debían haber } \\
\text { desarrollado alguna autonomía." }\end{array}$ \\
\hline
\end{tabular}

Cuadro 7 (continuación).- Análisis de los dilemas identificados en los Diarios de Clase y sus soluciones.

Los resultados y los datos presentados en este cuadro demuestran que la forma en que la profesora trató cada dilema fue diferente, constatándose que, al redactar los Diarios de Clase, la profesora reconoció las dificultades encontradas, los sentimientos que le eran inherentes y el modo como las resolvió y superó. Se puede destacar que esta concienciación contribuye al desarrollo del pensamiento metacognitivo de la profesora, esencial en la práctica de un profesor. Es de destacar también que el dilema Autonomía versus Dependencia surge en cuatro clases, siendo solventado de acuerdo con la situación/tarea en que surgió. Esta incidencia puede reflejar la preocupación de la profesora con el desarrollo de esta competencia.

\section{CONCLUSIÓN}

Este trabajo incidió en la aplicación de una planificación, centrada en la enseñanza por resolución de problemas, a un tema de Geología.

Los datos obtenidos a partir de los Diarios de Clase permiten afirmar que la profesora fue tomando conciencia de sus dificultades, intentando superarlas. Fueron varias las ventajas que se demostró estaban asociadas a este instrumento: i) al implicar escribir, favorece la organización del pensamiento reflexivo del profesor; ii) permite a la profesora establecer relaciones entre la experiencia actual y experiencias anteriores y iii) facilita la descripción de las tareas y de los estados emocionales relacionados con ellas. En este estudio la utilización de los diarios de clase presenta también algunas limitaciones que es importante que se tengan en cuenta en su futura utilización. La redacción de los acontecimientos muchas veces corresponde a una representación de lo real llena de subjetividad, lo que puede poner en cuestión la objetividad que se espera de una investigación. Se trata de una limitación típica de un estudio donde el profesor es también el investigador. Esta limitación puede ser, no obstante, minimizada a través de una mayor inclusión de datos reales, de un intercambio de informaciones obtenidas con los diarios y con otros instrumentos, como por ejemplo entrevistas, y de debates con otros investigadores. 
La utilización de los Snapshots en el estudio permitió verificar que se trató de instrumentos que demuestran la perspectiva del alumno referente al proceso de enseñanza y aprendizaje. Las respuestas de los alumnos permitieron una constante evaluación de la práctica lectiva. Sin embargo, y conforme se mencionó anteriormente, es fundamental trabajar más con los alumnos para que amplíen sus respuestas en las dimensiones $\mathrm{D}$ y $\mathrm{E}$ (dimensiones estrechamente relacionadas con procesos metacognitivos). En futuros estudios se podrá tratar de minimizar las limitaciones sentidas en estas dimensiones a través de un debate abierto y esclarecedor con los alumnos sobre el objetivo de estas dimensiones.

El análisis de los Snapshots y de los Diarios de Clase, permitió relatar y conceptualizar el proceso vivido, ya sea en lo que respecta a la enseñanza y al aprendizaje de los temas en cuestión, o desde el punto de vista del desarrollo de la profesora como docente, en una integración interactiva entre la teoría y la práctica esencial en la investigación educativa (Evans, 2004). Consideramos que podemos afirmar también que el proceso metodológico utilizado auxilió la introducción de mejoras en la práctica lectiva de la profesora, contribuyendo así a aumentar la calidad en el aprendizaje de los alumnos. Esta optimización del proceso de enseñanza y de aprendizaje se reflejó, por ejemplo, en el aumento de aspectos más positivos y la disminución de los aspectos menos positivos de la clase y dudas planteadas por los alumnos en los Snapshots de las últimas aulas. Las dimensiones C y D también fueron afectadas, verificándose un aumento de la complejidad de las respuestas de los alumnos.

El planeamiento fue ajustado al tipo de estrategias seleccionadas según la RP. Sería conveniente el recurso a un número más grande de clases para el logro eficaz de los objetivos definidos previamente.

Reseñar, finalmente, que la utilización de una enseñanza a través de Resolución de Problemas, fundamentado en resultados provenientes de la investigación, permitió el desarrollo de competencias en los alumnos que estudian asignaturas de ciencias. Principalmente, competencias relacionadas con el desarrollo de actitudes de curiosidad en relación a fenómenos naturales; de conocimientos susceptibles de una mejor compresión de esos fenómenos; de comunicación en ciencias y de cooperación con los demás en el proceso de crecimiento cognitivo.

\section{REFERENCIAS}

ANGELOPOULOS, V. y TSAPARLIS, G. (2000). A Model of Problem Solving: Its operation, validity and usefulness in the case of organic-synthesis problems. Journal of Science Education, 84, pp.131-153.

BROEKKAMP H., TACONIS; Y FERGUSON-HESSLER(2001). Teaching Science Problem Solving: An Overview of Experimental work. Journal of Research in Science Teaching, 38 (4), pp. 442-468.

COSTA, N. (coord.) (2002). O Ensino e a Aprendizagem do Som: Materiais para a sala de aula. Monografia, Aveiro: Departamento de Didáctica e Tecnologia Educativa da Universidade de Aveiro.

EVANS, L (2002). Reflective Practice in Educational Research. Developing Advanced Skills. Londres: Continuum. 
GARCIA, C. (1999). Formação de Professores - Para uma Mudança Educativa. Porto: Porto Editora.

GARDNER, J. (2003). Problem-Based learning. Acedido em 19 de Julho de 2004, em $<$ www.studygs.net $>$.

GARRET, R. M. (1995). Resolver Problemas en la enseñansa de las Ciências. Alambique, 5, pp. 6-15.

GIL PÉREZ, D. et al. (1999). Tiene Sentido Seguir Distinguiendo Ebtre Aprendizage de Conceptos, Resolución de problemas de Lápiz y Papel y Realización de Práticas de Laboratório. Enseñanza de las Ciências, 17 (2), pp.311-320.

LOPES, B. (2004). Aprender e Ensinar Física. Braga. Lisboa: Fundação Calouste Gulbenkian e Fundação para a Ciência e a Tecnologia.

MAREN, J. (1996). Méthodes de Recherche pour L'Éducation. Bruxelles: De Boeck Université.

MERTLER, G. (1997). Students as stakeholders in teacher evaluation: teachers perceptions of a formative feedback model. En "Annual Meeting of the MidWestern Educational Research Association", em Outubro. Acedido em 30 de Novembro de 2005, em www.eric.ed.gov.

NETO, A. (1995). Contributos para uma nova didáctica da resolução de problemas: um estudo de orientação metacognitiva em aulas de Física no ensino secundário. Tese de Doutoramento (não publicada). Universidade de Aveiro.

VALENTE, M.; SALEMA, M. H.; MORAIS, M.M. Y CRUZ, M.N. O. (1989). A metacognição. Revista de Educação, 1(3), pp. 47-52.

VIEIRA, F. (1993). Supervisão. Uma prática reflexiva de professores. Porto: Edições ASA.

ZABALZA, M. (1994). Diários de Aula. Porto: Porto Editora. 

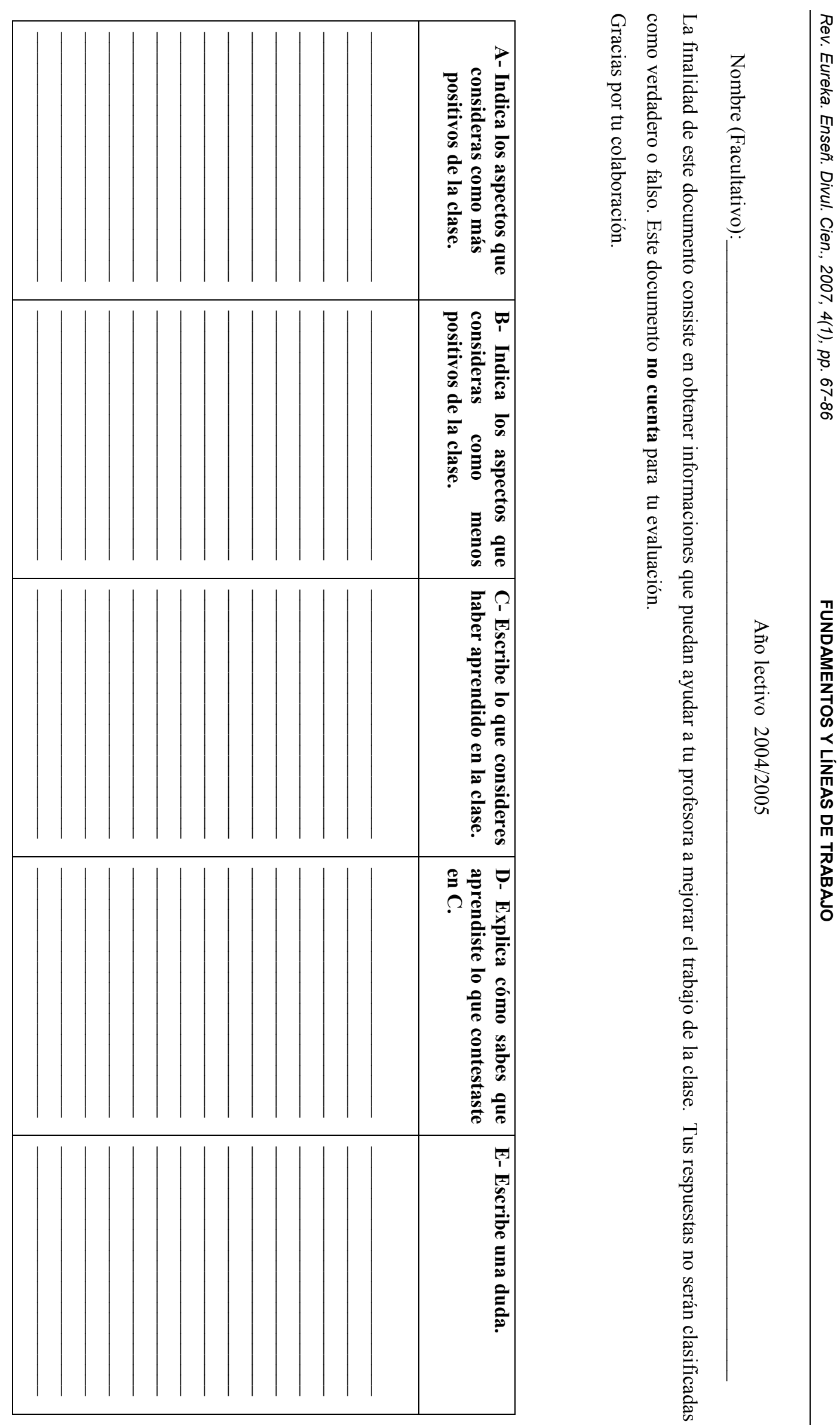


\section{SUMMARY}

This research study consisted on the conception, application and evaluation of a teaching planning based in a subject of Natural Sciences (Geology) and through Problem Solving. The planning took place in the natural context of the classroom, in a school of the North of Portugal, with students between 12 and 13 years old. The teacher of the students assumed the double role of teacher and researcher. It was used a methodology of Action Research, and it was used techniques of collection of data such us: diagnose tests, class diaries and Snapshots. The study allowed to understand the effectiveness of the education process and learning in the optimization of the quality of the educational practice, when this one is: (a) based on the investigation in Didactics of Sciences, specifically with regard to the investigation area on Problem Solving; (b) is directed by a teacher assumes both the teacher and the researcher roles; (c) is supported by researchers with active role in the researcher process.

Keywords: Problem Solving; Action Research; Class Diaries; Snapshot. 